\title{
Letter to the Editor: Determination of Any Correlation between Sagittal Spinopelvic Configuration and Progressive Collapse of Acute Osteoporotic Compression Spine Fractures: A Retrospective Radiological Analysis
}

\author{
Kalyan Kumar Varma Kalidindi, Kuldeep Bansal \\ Department of Spine Service, Indian Spinal Injuries Centre, New Delhi, India
}

To the editor,

We read with great interest, an article titled "Determination of any correlation between sagittal spinopelvic configuration and progressive collapse of acute osteoporotic compression spine fractures: a retrospective radiological analysis" by Smorgick et al. [1]. We congratulate the authors for their laudable work. They observed that spinopelvic configuration does not influence the progression of osteoporotic collapse. However, we seek clarification on a few concerns.

(1) The authors have mentioned "sagittal" spino-pelvic configuration in their title. However, they have analyzed only spinopelvic parameters including pelvic incidence, sacral slope and pelvic tilt. No sagittal parameters have been analyzed in the study.

(2) The authors have not considered the neurological status of the patient at the time of presentation in the selection criteria. They have mentioned that all the patients were treated with a spinal orthosis for 3 months. We would like to know if the patients who presented with neurological deterioration were also treated conserva- tively.

(3) There was no mention of the level of injury in the inclusion criteria. However, the authors have not mentioned how patients with cervical (e.g., odontoid fractures are common after a trivial fall in the elderly) and upper thoracic fractures were managed at their center.

(4) The authors mentioned that pathological fractures were excluded. However, they mentioned that patients were evaluated with and X-ray and computed tomography scan (in 90\% patients). We would like to know how the pathological fractures were diagnosed without using advanced imaging techniques such as magnetic resonance imaging (MRI) scan which are more sensitive in diagnosing them [2].

(5) The authors have mentioned including only acute fractures. However, X-rays may sometime be difficult to differentiate healed fractures from acute fractures [3]. MRI and bone scan revealing edematous lesions can help in diagnosing acute fractures. Also, since the trauma would be trivial, it is difficult to attribute the fracture as acute based on history alone. How did the authors confirm that the fractures are acute? 
(6) The authors have failed to mention the position in which radiographs were taken. Standing weight bearing radiographs could reveal more vertebral collapse as compared to lying down films. However, in acute fractures, it is difficult to perform standing radiographs when the patient presents with severe pain. Hence, the position of the patient when performing radiographs has an important role in this study where the vertebral body heights were measured. The authors may throw light on this aspect to the readers.

Again, we compliment the authors for their commendable work and hope that this study would benefit the readers.

\section{Conflict of Interest}

No potential conflict of interest relevant to this article was reported.

\section{References}

1. Smorgick Y, Geftler A, Goldstein S, Mirovsky Y, Blecher R, Anekstein Y. Determination of any correlation between sagittal spinopelvic configuration and progressive collapse of acute osteoporotic compression spine fractures: a retrospective radiological analysis. Asian Spine J 2020 Jan 8 [Epub]. https://doi. org/10.31616/asj.2019.0139.

2. Takigawa T, Tanaka M, Sugimoto $Y$, Tetsunaga T, Nishida K, Ozaki T. Discrimination between malignant and benign vertebral fractures using magnetic resonance imaging. Asian Spine J 2017;11:478-83.

3. Chen YJ, Lo DF, Chang CH, Chen HT, Hsu HC. The value of dynamic radiographs in diagnosing painful vertebrae in osteoporotic compression fractures. AJNR Am J Neuroradiol 2011;32:121-4. 